\title{
Classificação do Estado dos Olhos via Dados EEG e Redes Neurais Feed-Forward, Recorrente e Evolutiva
}

\author{
Danyellen Gonçalves*, Cristiano Garcia*, \\ Wilian Soares Lacerda*, Daniel Leite* \\ * Departamento de Automática, Universidade Federal de Lavras \\ Lavras, MG, Brasil (E-mails: danyellen.g@hotmail.com, \\ cristiano.garcia@ufla.br, lacerda@ufla.br,daniel.leite@ufla.br).
}

\begin{abstract}
Electroencephalography (EEG) data analysis has been widely used in a variety of application domains. This study aims at detecting the state of the eyes from 14 EEG electrodes. Three artificial neural models are briefly presented, computationally realized, and compared for the task of classifying the state of the eyes. A feed-forward Multi-Layer Perceptron (MLP) network, the recurrent Elman (ELM) network, and an evolving granular (EGNN) neural network are developed. Results show that the evolving EGNN approach outperforms the remaining approaches in terms of true positives and negatives regarding the state of the eye.

Resumo: A análise de dados de eletroencefalografia (EEG) tem sido largamente explorada em uma variedade de aplicações. O presente trabalho visa detectar o estado dos olhos a partir dos dados de 14 eletrodos EEG. Três modelos neurais artificiais são brevemente apresentados, implementados e comparados na tarefa de classificação do estado do olho. Empregou-se o modelo feed-forward Multi Layer Perceptron (MLP), o modelo recorrente de Elman (ELM), e o modelo granular evolutivo (EGNN). Os resultados mostram que a abordagem evolutiva EGNN supera as demais em termos de verdadeiros positivos e negativos com relação ao estado do olho.
\end{abstract}

Keywords: Electroencephalography; Pattern Classification; Evolving Intelligent Systems; Artificial Neural Networks; Data Stream

Palavras-chaves: Eletroencefalograma; Classificação de Padrões; Sistemas Inteligentes Evolutivos; Redes Neurais Artificiais; Fluxo de Dados

\section{INTRODUÇÃO}

Uma Interface Cérebro-Computador (do inglês BrainComputer Interface (BCI)) é um sistema de comunicação que permite que seres humanos enviem mensagens para o mundo externo sem passar pelas vias tradicionais dos nervos e músculos (Wolpaw et al., 2002). Com o advento desta tecnologia, surgiu a necessidade de classificação de padrões do pensamento, e também de modelos preditores robustos a partir de dados de eletrodos. As pesquisas na área de BCI vêm crescendo significativamente nas últimas décadas, impulsionadas por uma diversidade de aplicações, tais como controle de cadeira de rodas (Carlson and del R Millan, 2013), jogos de computador (Nijholt, 2008)(Marshall et al., 2013)(McMahan et al., 2015), sistemas de saúde e biomédicos (Pan et al., 2014)(Ossmy et al., 2011)(Sadatnezhad et al., 2011), rastreamento de emoções (Pham and Tran, 2012), e controle de dispositivos em casas inteligentes (Simoens et al., 2014).

\subsection{Classificação EEG do estado dos olhos}

Atualmente, existe uma demanda pela classificação de dados de eletroencefalografia (EEG) para detecção do estado dos olhos. O resultado destas pesquisas é de grande importância para avaliar o estado cognitivo do ser humano, tanto para assistência médica, como para algumas tarefas da vida diária. Por exemplo, a classificação de estado ocular através de sinais EEG foi aplicada com sucesso nas áreas de detecção do sono na direção de veículos (Yeo et al., 2009), detecção de crises epilépticas (Polat and Gunes, 2007), identificação do estado de alerta em bebês (Estévez et al., 2002), detecção do piscar do olho (Nguyen et al., 2013), controle de teclado virtual através de piscadas padronizadas (Chambayil et al., 2010), controle de cursor 2D (Trejo et al., 2006), entre outros.

Os sinais EEG que descrevem o estado dos olhos são dados providos continuamente como uma série temporal discreta, ver Figura 1. Várias abordagens de reconhecimento de padrões para séries temporais foram empregadas em estudos anteriores. Por exemplo, (Yeo et al., 2009) utilizou Maquinas de Vetor Suporte (SVM) para detectar sonolência durante a condução de um veículo a partir da frequência do piscar dos olhos. A referência (Sulaiman et al., 2011) empregou o método K-Nearest Neighbors (KNN) para identificação de estresse. Já em (Polat and Gunes, 2007), foi apresentado um sistema hibrido baseado em Arvore de Decisão e Transformada Rápida de Fourier (FFT) para detecção de crises epilépticas. Todos esses trabalhos mostraram que métodos de aprendizado de máquina e métodos estatísticos são interessantes na resolução de problemas de 


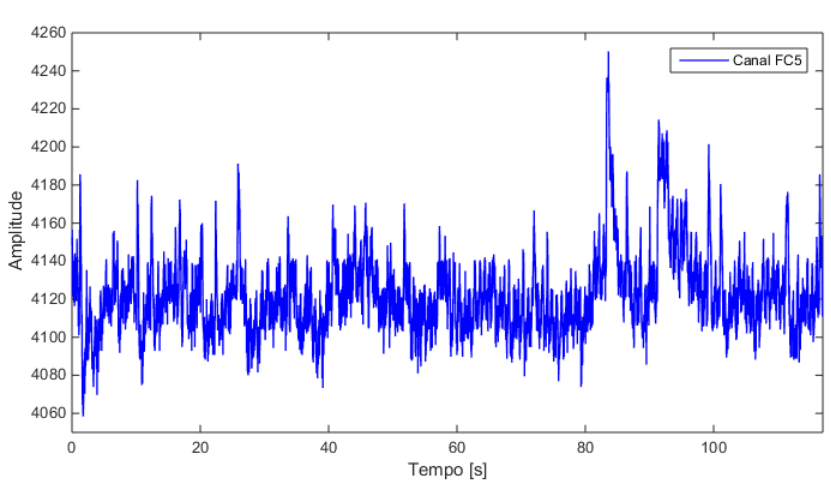

Figura 1. Exemplo de uma série temporal proveniente de um canal EEG em um experimento de detecção do estado do olho

classificação de dados sequenciais visando a identificação do estado dos olhos.

No presente trabalho, propomos a implementação e comparação de três modelos de redes neurais artificiais para classificação do estado dos olhos. Os modelos considerados usam diferentes arquiteturas. O primeiro modelo consiste de uma rede Multi Layer Perceptron (MLP) com uma camada intermediária e treinada via algoritmo de otimização de Levenberg-Marquardt (rede neural feedforward). O segundo modelo é uma rede recorrente de Elman (ELM) com uma camada intermediária. A saída dos neurônios da camada intermediária é conectada à entrada dos mesmos neurônios. Tal retro-alimentação retém uma cópia dos valores anteriores e a usa no processamento da amostra atual. Logo, a rede ELM mantém um estado de memória útil para processamento de dados temporais (Elman, 1990). Além disso, empregou-se uma rede neural granular evolutiva (EGNN). Esta rede usa um algoritmo de aprendizado incremental online e, portanto, não necessita de uma fase de treinamento offline e da estipulação prévia de uma estrutura. Seu algoritmo de aprendizado desenvolve a estrutura e os parâmetros do modelo EGNN a medida que os dados são disponibilizados. Trata-se de uma rede modular, pois se granula os dados de entrada em objetos fuzzy. Modelos locais são desenvolvidos e usados para estimar o estado do olho.

O artigo está organizado da seguinte forma. A seção 2 apresenta uma breve introdução às três abordagens neurais supracitadas. A seção 3 consiste de detalhes da base de dados do estado do olho e da metodologia utilizada para avaliação dos modelos. Os resultados comparativos são apresentados na seção 4 . A seção 5 conclui o trabalho.

\section{SOBRE MODELOS NEURAIS ARTIFICIAIS}

\subsection{Rede feed-forward $M L P$}

A rede neural MLP é o modelo estático feed-forward mais utilizado. A sua arquitetura é pré-selecionada. O fluxo da informação através da rede se dá em um único sentido, da camada de entrada para a camada de saída, como mostra a Fig. 2 (Koskela et al., 1996). Cada neurônio calcula sua saída com base na soma ponderada de todas as suas entradas multiplicadas pelos pesos das conexões de entrada. O neurônio usa uma função de ativação. Existem diferentes tipos de funções de ativação, como limiar, linear por partes, sigmóide, tangente-hiperbólica, Gauss. Este trabalho a função utilizada tanto na camada intermediária quanto na camada de saída é a função tangente-hiperbólica. Fixamos o número de camadas em 3 e avaliamos diferentes quantidades de neurônios intermediários.

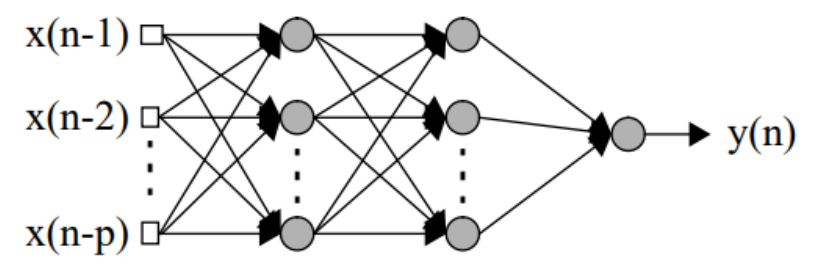

Figura 2. Esquema fundamental de um modelo neural MLP

A rede MLP é treinada ajustando-se os pesos das conexões entre neurônios. O método mais comum de treinamento de uma rede MLP consiste no uso do algoritmo de retropropagação dos erros de estimação (BP). Entretanto, apesar de ser usado com sucesso em vários tipos de problemas, esse algoritmo baseado em gradiente descendente possui convergência lenta, baixa estabilidade, e dificuldade para escapar de mínimos locais e plateaus. Neste trabalho é utilizado o algoritmo Levenberg-Marquart (LM) ou Mínimos Quadrados Amortecido, que utiliza uma técnica de otimização numérica e, geralmente, apresenta convergência mais rápida e eficiente do que o algoritmo $\mathrm{BP}$ a partir da variação de um coeficiente de amortecimento ao longo do treinamento (Gill and Murray, 1978).

\subsection{Rede recorrente ELM}

Uma rede neural com ao menos uma conexão de realimentação é chamada de rede recorrente. A rede de Elman é um tipo de rede com recorrência interna (Elman, 1990). A estrutura da rede ELM consiste de quatro camadas, i.e., camada de entrada, camada intermediária, camada de contexto, e camada de saída, conforme mostrado no esquema geral da Fig. 3. Para cada neurônio na camada intermediária existe uma unidade neural e conexão adicional conhecida como elementos de contexto. A idéia é conectar a saída de um neurônio intermediário à sua própria entrada. Isso significa que a rede possui uma memória do estado anterior construída através de feedback positivo. Esse feedback permite que a rede ELM aprenda padrões temporais. Os valores da camada de saída não são realimentados neste trabalho (Hayat, 2007).

\subsection{Rede granular evolutiva EGNN}

Uma rede neural granular evolutiva (EGNN) para modelagem on-line de sistemas não lineares e não estacionários foi proposta em Leite et al. (2013). Esta rede utiliza uma abordagem de aprendizado que gradualmente adapta sua estrutura - grânulos de informação e neurônios fuzzy, i.e., neurônios que implementam operadores de agregação fuzzy - bem como os parâmetros associados utilizando o fluxo de dados (paradigma evolutivo, ver Skrjanc et al. (2019)). A rede EGNN desenvolve modelos globais usando um algoritmo incremental, de passo único sobre os dados. Em outras palavras, o algoritmo recebe os dados e os 


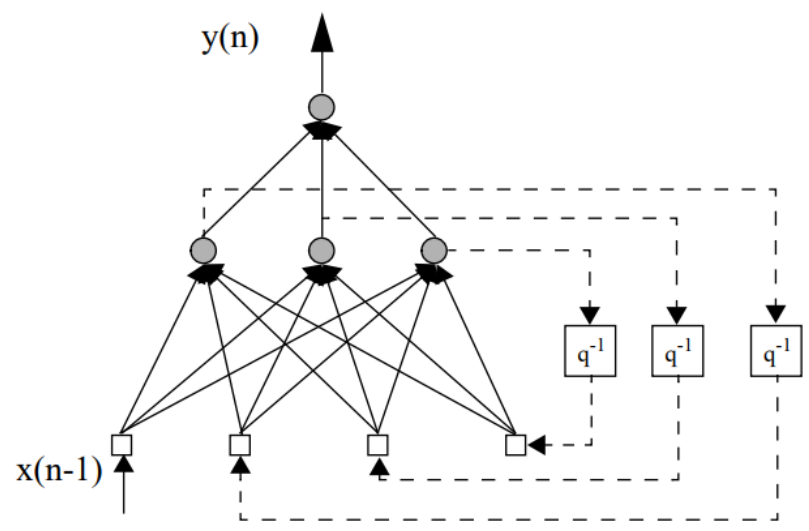

Figura 3. Esquema fundamental de um modelo neural ELM

processa apenas uma vez (uma época de treinamento). Além disso, EGNN não necessita de conhecimento a priori sobre o sistema em questão e sobre os dados, tais como distribuição de probabilidade e faixa de valores. A rede inicia o aprendizado assim que a primeira amostra é fornecida.

Originalmente, EGNN foi proposta como um classificador evolutivo. Ela superou o desempenho de modelos nãolineares alternativos em problemas clássicos de reconhecimento de padrões, como Iris e Wine (Leite et al., 2009). Em Leite et al. (2010) foi proposta uma versão semisupervisionada de EGNN para classificação de dados parcialmente rotulados. Neste artigo mostraremos apenas a estrutura da rede para classificação de padrões devido a restrição de espaço, e para um balanceamento da apresentação com relação a apresentação das redes MLP e ELM. Refira-se a Leite et al. (2013) para uma completa descrição de suas características e do algoritmo de aprendizado online a partir de fluxos de dados.

Em essência, seja $x=\left(x_{1}, \ldots, x_{n}\right)$ um vetor de entrada e $y$ a variável de saída. Considere que o fluxo de dados $(x, y)^{[h]}$, $h=1, \ldots$, seja medido a partir de uma função desconhecida $f$ que potencialmente pode ser não-linear e variante no tempo. Entradas $x_{j}$ e saída $y$ são dados numéricos (valores reais provenientes dos canais dos eletrodos EEG) neste trabalho.

A Fig. 4 mostra o esquema geral de um modelo EGNN. É importante notar que, inicialmente, neurônios e conexões não existem. A rede é desenvolvida em tempo real a medida que o fluxo de dados é disponibilizado. Logo, a estrutura EGNN mostrada na figura retrata um estágio posterior de evolução. A camada de entrada recebe $x^{[h]}$. A camada granular consiste de um conjunto de grânulos $G_{j}^{i}, j=1, \ldots, n ; i=1, \ldots, c$, estratificado a partir dos dados de entrada. Conjuntos fuzzy $G_{j}^{i}, i=1, \ldots, c$, formam uma partição fuzzy do $j$-ésimo domínio de entrada, $X_{j}$. Um grânulo $G^{i}=G_{1}^{i} \times \ldots \times G_{n}^{i}$ é uma relação fuzzy, i.e., um conjunto fuzzy multi-dimensional em $X_{1} \times \ldots \times X_{n}$. Dessa forma, $G^{i}$ possui função de pertinência $G^{i}(x)=$ $\min \left\{G_{1}^{i}\left(x_{1}\right), \ldots, G_{n}^{i}\left(x_{n}\right)\right\}$ em $X_{1} \times \ldots \times X_{n}$. O grânulo $G^{i}$ pode ter uma função local $p^{i}$ associada. Para classificação, é utilizada uma função de ordem 0 ,

$$
p^{i}\left(\hat{x}_{1}, \ldots, \hat{x}_{n}\right)=\hat{C}^{i}
$$

onde $\hat{C}^{i}$ é a classe estimada.

A camada de agregação é composta por neurônios fuzzy $A^{i}, i=1, \ldots, c$. Um neurônio fuzzy $A^{i}$ combina graus de similaridade ponderados $\left(\widetilde{x}_{1}^{i} w_{1}^{i}, \ldots, \widetilde{x}_{n}^{i} w_{n}^{i}\right)$ em um único valor $o^{i}$, que é o grau de ativação da regra $R^{i}$. A camada de saída processa $\left(o^{1}, \ldots, o^{c}\right)$ usando um neurônio fuzzy $A^{f}$. $A^{f}$ utiliza a S-norma max neste trabalho. A classe $\hat{C}^{i *}$ associada à regra mais ativa $R^{i *}$ é a saída da rede.

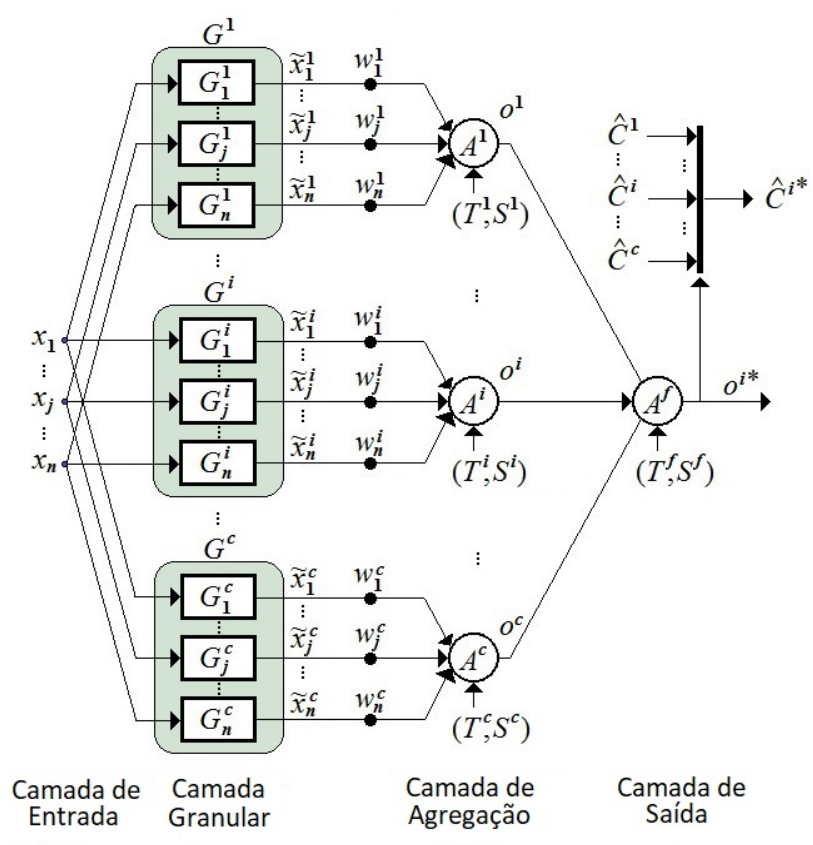

Figura 4. Rede neural granular evolutiva

Assumindo pesos e tipos de neurônios específicos, regras fuzzy extraídas do modelo EGNN são do tipo

$$
R^{i}: \operatorname{SE}\left(x_{1} \text { é } G_{1}^{i}\right) \mathrm{E} \ldots \mathrm{E}\left(x_{n} \text { é } G_{n}^{i}\right) \operatorname{ENTÃO}\left(\hat{y} \text { é } \hat{C}^{i}\right) \text {. }
$$

Como: (i) os conjuntos fuzzy $G_{j}^{i} \forall i, j$, são variantes no tempo; (ii) uma diversidade de funções de agregação podem ser usadas no corpo neural; e (iii) grânulos fuzzy se sobrepõem no espaço de entrada, então a superfície de separação de classes provida por um modelo EGNN é nãoestacionária e pode ser altamente não-linear.

\section{METODOLOGIA}

\subsection{Sobre a base de dados}

O conjunto de dados utilizado neste trabalho foi gerado e apresentado em (Rösler and Suendermann, 2013) a partir de um experimento real. Os dados foram doados para a Universidade da Califórnia em Irvine (UCI Machine Learning Repository) que os disponibiliza publicamente. Os sinais EEG foram derivados de uma medição contínua por 117 segundos utilizando o Neuro-headset Emotiv Epoc EEG com 14 eletrodos à seco localizado nas posições F7, F8, F3, F4, FC5, FC6, T7, T8, P3, P4, P7, P8, O1 е O2, de acordo com o Sistema Internacional 10-20, vide Fig. 5. Um sumário da base de dados é apresentado no gráfico da 
Fig. 6 para uma noção geral do comportamento dos sinais provenientes dos canais EEG durante o experimento de monitoramento do estado dos olhos.
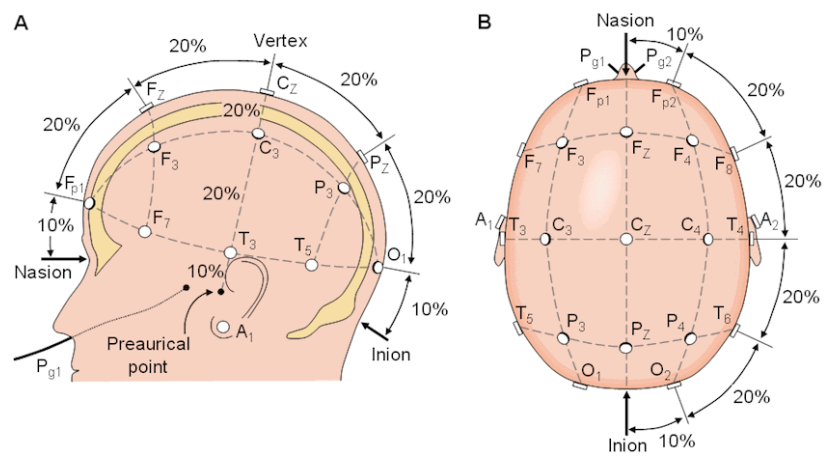

Figura 5. Posicionamento de eletrodos de acordo com o Sistema Internacional 10-20

O estado dos olhos de uma pessoa foi detectado por meio de uma câmera, durante a medição dos sinais EEG, e adicionado manualmente à base de dados após a análise do vídeo (valor de saída desejado). Para a variável de saída, o valor ' 1 ' indica o estado do olho fechado, e '0' se refere ao estado do olho aberto. As amostras são disponibilizadas na ordem cronológica. Em outras palavras, as amostras são indexadas no tempo.

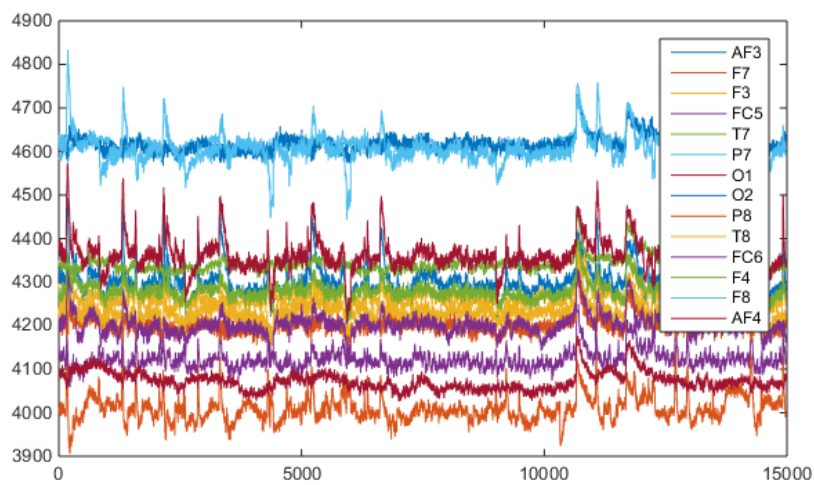

Figura 6. Base de dados EEG para classificação do estado dos olhos

Em particular, nesta base de dados há quatro amostras, viz. 899, 10387, 11510 e 13180, contendo erros visíveis. Os valores são maiores do que aqueles valores permitidos pelos canais EEG. Estes dados foram considerados outliers. Visto que o número de amostras com erros é significativamente menor do que a quantidade total de amostras, optou-se por excluir as amostras em questão, essencialmente. A distorção de valores de covariância entre atributos é desprezível neste caso. Portanto, apenas 14976 amostras são utilizadas nos experimentos, sendo 6722 relativas ao estado do olho fechado, e 8254 referentes ao estado do olho aberto. Para os modelos offline MLP e ELM, 70\% dos dados foram utilizados para treinamento, e 30\% para teste. Para o caso do modelo online EGNN, os dados são apresentados apenas uma vez, como um fluxo online. As fases de treinamento e teste ocorrem concomitantemente, amostra por amostra. Primeiro provê-se uma estimação, em seguida ocorre o aprendizado.

\subsection{Sobre modelos neurais, software e hardware}

Diferentes configurações de modelos neurais MLP e ELM, i.e., considerando diferentes números de neurônios na camada intermediária, assim como o modelo evolutivo EGNN, são comparados em termos de acurácia na classificação dos padrões 'olho aberto' e 'olho fechado'. Para todas as arquiteturas de rede, o número de neurônios de entrada é igual ao número de canais EEG, i.e., 14. Apenas um neurônio de saída é considerado.

As funções 'patternnet' e 'train' são usadas para a construção de modelos feed-forward. Os neurônios intermediários e de saída usam funções de ativação do tipo tangente-hiperbólica. Investigou-se arquiteturas MLP com até 30 neurônios. Arquiteturas maiores demandam um tempo de treinamento substancial via algoritmo Levenberg-Marquardt. A rede recorrente ELM também contém neurônios implementando funções tangentehiperbólica. Os neurônios de contexto são conectados aos neurônios intermediários em retro-alimentação. Similarmente, investigou-se arquiteturas com até 30 neurônios. O algoritmo Gradiente Descendente é usado no treinamento. O critério de parada de ambos os algoritmos é o número de épocas, 2000. O método de validação cruzada holdout é utilizado, no qual $70 \%$ das amostras da base de dados são usadas para treinamento, e o restante para teste dos modelos.

EGNN usa os parâmetros iniciais $\rho=0,25, \eta=2 \mathrm{e}$ $h_{r}=300$ (ver detalhes em (Leite et al., 2013)). Fundamentalmente, $\rho$ é a granularidade máxima, e $\eta$ e $h_{r}$ são limiares úteis para mescla e remoção de grânulos da rede neural ao longo do aprendizado online. Geralmente, se tais parâmetros são escolhidos dentro das faixas [0,1 0,4], [2 3] e [200 500], respectivamente, o algoritmo produz estruturas ligeiramente maiores ou menores, mas efetivas no problema de deteção do estado do olho. O número de neurônios e grânulos é imprevisível a priori; ele varia dependendo da sequência de movimento do olho.

A acurácia dos classificadores é calculada conforme,

$$
A c c(\%)=\frac{V P+V N}{V P+F P+V N+F N},
$$

onde $V P, F P, V N$ e $F N$ se referem a verdadeiros e falsos, positivos e negativos. A classe negativa (classe 0) indica o estado do olho aberto. A classe positiva (classe 1) refere-se ao estado do olho fechado. Os experimentos foram realizados no ambiente Matlab R2014b usando um processador Intel Core i5-7200U 2.70GHz com 8Gb de RAM. O tempo de processamento dos dados e adaptação dos diferentes modelos é comparado.

\section{RESULTADOS}

Um sumário dos resultados de treino e teste dos modelos MLP e ELM para classificação do estado dos olhos é apresentado nas Tabelas 1 e 2 . Variou-se a quantidade de neurônios na camada intermediária, entre 1 e 15.

Nota-se nas Tabelas 1 e 2 que quanto maior o número de neurônios intermediários, maior a acurácia de treino e teste de ambas as abordagens, feed-forward e recorrente. Entretanto, o tempo necessário para o treinamento offline das 
redes começa a se tornar excessivo. As melhores estruturas MLP e ELM usam 30 neurônios. Nesse caso, a rede MLP obteve um desempenho online de $91,9 \%$ contra $85,7 \%$ da rede ELM. Apesar de a rede ELM possuir mais parâmetros e, portanto, um maior potencial de alcançar fronteiras de separação de classes mais precisas, há uma maior dificuldade na busca por mínimos locais interessantes no espaço dos parâmetros já que ele possui maior dimensionalidade. Nota-se que todos os modelos gerados apresentam boa capacidade de generalização do comportamento observado durante o processo de treinamento.

A rede neural granular evolutiva EGNN foi desenvolvida em modo online de acordo com o fluxo de dados. A rede obteve acurácia de classificação de $95,7 \%$ para a base de dados de teste. O número médio de regras SE-ENTÃO ao longo das 14976 iterações foi de 7,23 $\pm 5,2$. Além disso, EGNN gastou 6,6 segundos para ser desenvolvida, i.e., aproximadamente $1 \%$ do tempo de construção de modelos MLP, e 5\% para o caso de modelos ELM, considerando suas configurações com melhor desempenho.

As matrizes de confusão para os melhores modelos MLP e ELM obtidos, e para o modelo EGNN são mostradas nas Figs. 7, 8 e 9. Nota-se que, enquanto os modelos offline, MLP e ELM, tiveram uma maior dificuldade com relação a detecção da classe desejada ' 1 ' (olho fechado), estimando a saída '0' (olho aberto), a rede evolutiva EGNN não teve esta dificuldade. EGNN equilibrou a quantidade de falsos positivos e negativos a partir da evolução de sua estrutura e parâmetros em modo online. Situações diferentes, surgindo nos dados dos canais EEG, conforme mostrado na Fig. 6, puderam ser capturadas a partir da criação e do desenvolvimento de novos modelos locais.

Tabela 1. Acurácia dos modelos neurais feedforward multi-camadas - MLP

\begin{tabular}{l|l|l|l}
\hline \# Total Neurônios & Treino & Teste & Tempo (seg) \\
\hline \hline 16 & $68,1 \%$ & $68,2 \%$ & 59 \\
18 & $80,7 \%$ & $80,1 \%$ & 363 \\
20 & $84,9 \%$ & $84,9 \%$ & 397 \\
23 & $90,8 \%$ & $89,5 \%$ & 451 \\
25 & $92,8 \%$ & $90,4 \%$ & 456 \\
30 & $93,1 \%$ & $91,9 \%$ & 621 \\
\hline
\end{tabular}

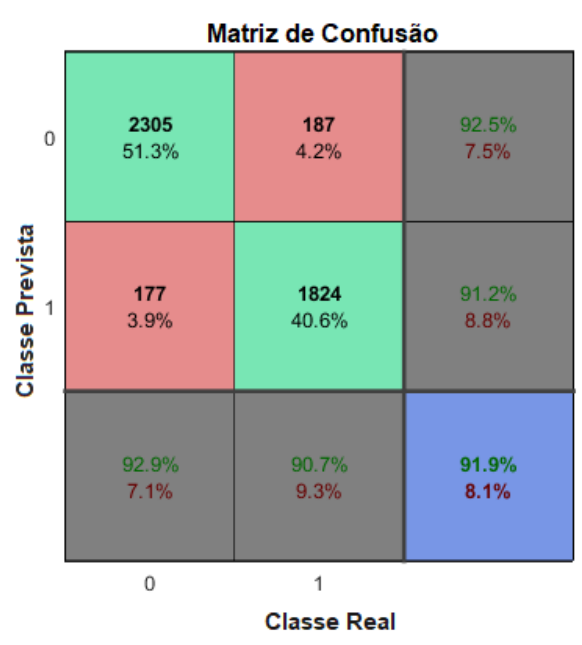

Figura 7. Matriz de confusão do melhor modelo feedforward MLP, com 30 neurônios intermediários
Tabela 2. Acurácia dos modelos neurais recorrentes de Elman - ELM

\begin{tabular}{l|l|l|l}
\hline \# Total Neurônios & Treino & Teste & Tempo (seg) \\
\hline \hline 16 & $75,5 \%$ & $76,5 \%$ & 77 \\
18 & $77,1 \%$ & $76,6 \%$ & 66 \\
20 & $76,1 \%$ & $76,9 \%$ & 87 \\
23 & $78,7 \%$ & $78,9 \%$ & 98 \\
25 & $82,9 \%$ & $83,4 \%$ & 108 \\
30 & $85,1 \%$ & $85,7 \%$ & 117 \\
\hline
\end{tabular}

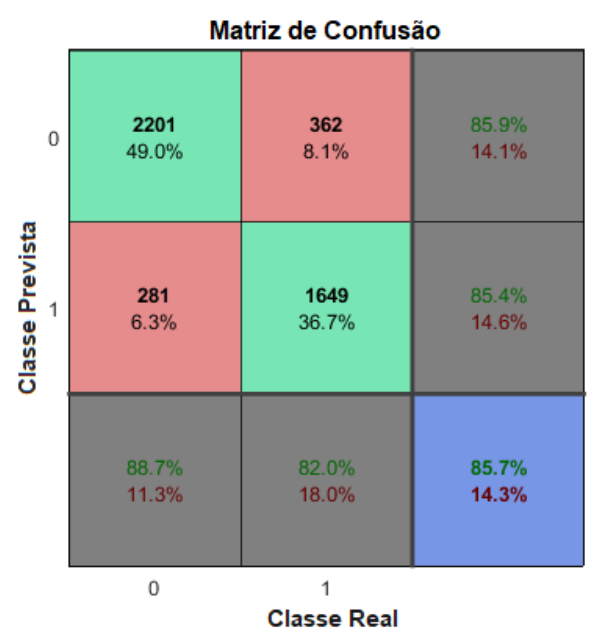

Figura 8. Matriz de confusão do melhor modelo recorrente ELM, com 30 neurônios intermediários

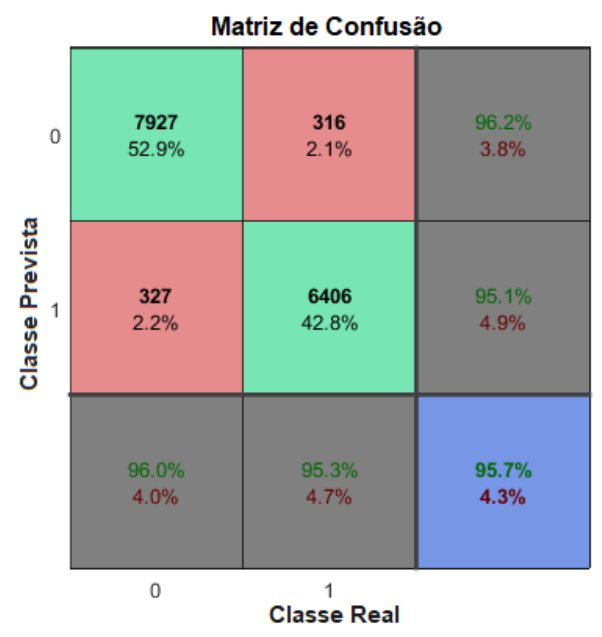

Figura 9. Matriz de confusão do modelo granular evolutivo EGNN, com 7,23 elementos locais de processamento em média

\section{CONCLUSÃO}

Este estudo apresenta três tipos de modelos neurais desenvolvidos para um problema de detecção do estado do olho a partir de dados EEG. Os dados são gerados a partir de 14 eletrodos à seco do dispositivo Emotiv. Desenvolveram-se redes feed-forward MLP, redes recorrentes de Elman ELM, e redes granulares evolutivas EGNN.

O estudo comparativo considerando experimentos computacionais mostrou que o modelo evolutivo EGNN é mais eficiente na classificação dos sinais temporais EEG (95,7\% 
de acurácia contra 91,9\% e 85,7\% de MLP e ELM, respectivamente) devido à sua habilidade de capturar a essência da informação no fluxo de dados, e se adaptar parametricamente e estruturalmente ao longo do tempo. Em termos de tempo de processamento e adaptação de parâmetros, é notável que EGNN é capaz de lidar com dados em uma ordem de grandeza inferior a modelos offline, como MLP e ELM. Enquanto os últimos requerem aproximadamente 10 e 2 minutos para lidar com as amostras em questão, EGNN obteve seu melhor desempenho em aproximadamente 6 segundos. Logo, este modelo neural, juntamente com seu algoritmo de aprendizado incremental, é um candidato interessante para problemas que envolvem grandes quantidades de amostras e atributos, e que potencialmente podem mudar ao longo do tempo.

Em trabalhos futuros será considerada a criação de bases de dados EEG próprias e problemas de classificação multiclasse usando métodos neurais granulares evolutivos. Além disso, serão considerados problemas de controle de dispositivos físicos a partir da rotulação de grânulos e clusters com sinais de atuação.

\section{AGRADECIMENTOS}

Este trabalho recebeu apoio do Instituto Serrapilheira, número do processo Serra - 1812-26777. A primeira autora agradece à Fundação de Amparo à Pesquisa do Estado de Minas Gerais (FAPEMIG) pela bolsa de mestrado. O quarto autor agradece à FAPEMIG, processo APQ-0338418.

\section{REFERÊNCIAS}

Carlson, T. and del R Millan, J. (2013). Brain-controlled wheelchairs: a robotic architecture. IEEE Robotics and Automation Magazine, 20(1), 65-73.

Chambayil, B., Rajesh, S., and Rameshwar, J. (2010). Virtual keyboard BCI using Eye blinks in EEG. Wireless and Mobile Computing Networking and Communications (WiMob) 2010 IEEE 6th International Conference.

Elman, J.L. (1990). Finding structure in time. Cognitive science, 14(2), 179-211.

Estévez, P.A., Held, C.M., Holzmann, C.A., Perez, C.A., Pérez, J.P., Heiss, J., Garrido, M., and Peirano, P. (2002). Polysomnographic pattern recognition for automated classification of sleep-waking states in infants. Medical and Biological Eng and Comput, 40(1), 105-113.

Gill, P.E. and Murray, W. (1978). Algorithms for the solution of the nonlinear least-squares problem. SIAM Journal on Numerical Analysis, 15(5), 977-992.

Hayat, M. (2007). Short term load forecasting using artificial neural networks for the west of Iran. Journal of Applied Sciences, 12, 1582-1588.

Koskela, T., Lehtokangas, M., Saarinen, J., and Kaski, K. (1996). Time series prediction with multilayer perceptron, FIR and Elman neural networks. Proceedings of the World Congress on Neural Networks, 491-496.

Leite, D., Costa, P., and Gomide, F. (2009). Evolving granular classification neural networks. In IEEE International Joint Conf on Neural Networks, 1736-1743.

Leite, D., Costa, P., and Gomide, F. (2010). Evolving granular neural network for semi-supervised data stream classification. In The 2010 international joint conference on neural networks (IJCNN), 1-8. IEEE.
Leite, D., Costa, P., and Gomide, F. (2013). Evolving granular neural networks from fuzzy data streams. Neural Networks, 38, 1-16.

Marshall, D., Coyle, D., Wilson, S., and Callaghan, M. (2013). Games, gameplay, and BCI: the state of the art. Computational Intelligence and Games, CIG'08. IEEE Symposium On, 5, 82-99.

McMahan, T., Parberry, I., and Parsons, T. (2015). Modality specific assessment of video game player's experience using the Emotiv. Entertainment Comput, 7, 1-6.

Nguyen, T., Nguyen, T.H., Truong, K.Q.D., and van Vo, T. (2013). A mean threshold algorithm for human eye blinking detection using EEG. 4th Int Conf on the Development of Biomedical Eng, 275-279.

Nijholt, A. (2008). BCI for games: A 'state of the art' survey. Int Conf Entertainment Comput, 225-228.

Ossmy, O., Tam, O., Puzis, R., Rokach, L., Inbar, O., and Elovici, Y. (2011). MindDesktop-Computer Accessibility for Severely Handicapped. ICEIS, 4, 316-320.

Pan, J., Xie, Q., He, Y., Wang, F., Di, H., Laureys, S., Yu, R., and Li, Y. (2014). Detecting awareness in patients with disorders of consciousness using a hybrid braincomputer interface. Journal of Neural Eng, 11(5), 11p.

Pham, T.D. and Tran, D. (2012). Emotion recognition using the Emotiv Epoc device. International Conference on Neural Information Processing, 394-399.

Polat, K. and Gunes, S. (2007). Classification of epileptiform EEG using a hybrid system based on decision tree classifier and fast Fourier transform. Applied Mathematics and Computation, 187(2), 1017-1026.

Rösler, O. and Suendermann, D. (2013). First step towards eye state prediction using EEG. ICAIHMLS.

Sadatnezhad, K., Boostani, R., and Ghanizadeh, A. (2011). Classification of BMD and ADHD patients using their EEG signals. Expert Syst Appl, 38, 1956-1963.

Simoens, P., de Coninck, E., Vervust, T., Wijmeersch, J.F.V., Ingelbinck, T., Verbelen, T., de Beeck, M.O., and Dhoedt, B. (2014). Vision: smart home control with head-mounted sensors for vision and brain activity. Proceedings of the fifth International Workshop on Mobile Cloud Computing \& Services, 29-33.

Skrjanc, I., Iglesias, J., Sanchis, A., Leite, D., Lughofer, E., and Gomide, F. (2019). Evolving fuzzy and neuro-fuzzy approaches in clustering, regression, identification, and classification: A survey. Info Sci, 490, 344-368.

Sulaiman, N., Taib, M.N., Lias, S., Murat, Z.H., Aris, S.A.M., and Hamid, N.H.A. (2011). Novel methods for stress features identification using EEG signals. International Journal of Simulation: Systems, Science and Technology, 12, 27-33.

Trejo, L., Rosipal, R., and Matthews, B. (2006). Braincomputer interfaces for 1-D and 2-D cursor control: designs using volitional control of the EEG spectrum or steady-state visual evoked potentials. IEEE Trans Neural Syst Rehabil Eng, 14(2), 225-229.

Wolpaw, J.R., Birbaumer, N., McFarland, D.J., Pfurtscheller, G., and Vaughan, T.M. (2002). Brain-computer interfaces for communication and control. Clinical neurophysiology, 113(6), 767-791.

Yeo, M.V., Li, X., Shen, K., and Wilder-Smith, E.P. (2009). Can SVM be used for automatic EEG detection of drowsiness during car driving? Safety Science, 47, $115-124$. 